\title{
Conformational changes in glycine tri- and hexapeptide
}

\author{
Alexander V. Yakubovitch, Ilia A. Solov'yov*, Andrey V. Solov'yov ${ }^{\dagger}$, and Walter Greiner \\ Frankfurt Institute for Advanced Studies, \\ Max von Laue Str. 1, 60438 Frankfurt am Main, Germany
}

\begin{abstract}
We have investigated the potential energy surfaces for glycine chains consisting of three and six amino acids. For these molecules we have calculated potential energy surfaces as a function of the Ramachandran angles $\varphi$ and $\psi$, which are widely used for the characterization of the polypeptide chains. These particular degrees of freedom are essential for the characterization of proteins folding process. Calculations have been carried out within ab initio theoretical framework based on the density functional theory and accounting for all the electrons in the system. We have determined stable conformations and calculated the energy barriers for transitions between them. Using a thermodynamic approach, we have estimated the times of the characteristic transitions between these conformations. The results of our calculations have been compared with those obtained by other theoretical methods and with the available experimental data extracted from the Protein Data Base. This comparison demonstrates a reasonable correspondence of the most prominent minima on the calculated potential energy surfaces to the experimentally measured angles $\varphi$ and $\psi$ for the glycine chains appearing in native proteins. We have also investigated the influence of the secondary structure of polypeptide chains on the formation of the potential energy landscape. This analysis has been performed for the sheet and the helix conformations of chains of six amino acids.
\end{abstract}

\section{INTRODUCTION}

It is well known that proteins consist of amino acids whose number may vary in the range from hundreds up to tens of thousands. Small fragments of proteins are usually called polypeptide chains or polypeptides. This work is devoted to a study of the conformational

\footnotetext{
* On leave from the A.F. Ioffe Institute, St. Petersburg, Russia. E-mail: ilia@th.physik.uni-frankfurt.de

$\dagger$ On leave from the A.F. Ioffe Institute, St. Petersburg, Russia. E-mail: solovyov@fias.uni-frankfurt.de
} 
properties of glycine polypeptide chains.

Since recently, it became possible to study experimentally small fragments of proteins and polypeptides in the gas phase with the use of the MALDI mass spectroscopy [1, 2, 3, 4] and the ESI mass spectroscopy [5, 6]. From theoretical view point, investigation of small polypeptides is of significant interest because they can be treated by means of ab initio methods allowing accurate comparison of theoretical predictions with experiment. The results of $a b$ initio calculations can be then utilized for the development of model approaches applicable for the description of larger and more complex protein structures.

Polypeptides are characterized by the primary and the secondary structure [7, 8, 9, 10]. Different geometrical configurations of a polypeptide are often called as the conformations. One can expect that chemical and physical properties of various conformations of complex molecules might differ significantly. The number of various conformations (isomeric states) grows rapidly with the growth of a system size. Thus, a search for the most stable conformations becomes an increasingly difficult problem for large molecules. With the help of the NMR spectroscopy and the X-rays diffraction analysis it has been shown [9], that the sheet and the helix structures are the most prominent elements of the protein secondary structure.

The main difference between the sheet and the helix structures is due to the difference of the dihedral angles formed by the atoms of the polypeptide chains in the two cases. These degrees of freedom are responsible for the transition of the molecule from one conformation to another. By increasing the temperature of the system, the degrees of freedom responsible for twisting of the polypeptide chain can be activated. The study of this transition and evaluation of its characteristic duration are of significant interest, because this problem is closely related to one of the most intriguing problems of the protein physics - the protein folding. To study this transition it is necessary to investigate the potential energy surface of amino acid chains with respect to their twisting. Besides the protein folding, the potential energy landscapes of polypeptides carry a lot of detail and useful information about the structure of these molecules.

In the present paper we have studied the potential energy surface for small glycine chains. These molecules were chosen because they are often present in native proteins as fragments, and also because they allow ab initio theoretical treatment due to their relatively small size.

Previously, only glycine and alanine dipeptides were studied in detail. Sometimes their analogues were used to reduce the computational costs (for example (S)- $\alpha$ - 
(formylamino)propanamide). In refs. 11, 12, 13] alanine and glycine dipeptides were investigated within the Hartree-Fock theory. In these papers the potential energy surfaces were calculated versus the twisting angles of the molecules. Different stable conformations of the dipeptides, corresponding to different molecular conformations, were determined. Each stable conformation of the molecule was additionally studied on the basis of the perturbation theory, which takes into account many-electron correlations in the system. In refs. [14, 15, 16, 17, 18, 19] different conformations and their energies were determined within the framework of the density functional theory. In ref. [19] dynamics of the alanine dipeptide analog was discussed and time of the transition between the two conformations of the alanine dipeptide was found. In [20] the glycine molecule was studied with accounting for the many electron correlations within the many-body perturbation theory. In this work the most important glycine isomers and the frequencies of the normal vibration modes were determined.

A number of papers were devoted to the study of tripeptides. In refs. [21, 22, 23, 24, 25] dynamics of the alanine and glycine tripeptides was studied by means of classical molecular dynamics and with the use of semi-empirical potentials (such as GROMOS, CHARMM and AMBER). In ref. [26] within the framework of the Hartree-Fock theory several stable conformations of alanine and glycine tripeptides were found. In ref. [27] the Raman and IR spectra for alanine and glycine tripeptides were measured in neutral, acidy and alkali environments.

Polypeptides have been studied less. We are aware of only several related papers. In particular, stable conformations of neutral and charged alanine hexapeptides were obtained with the use of empirical potentials and discussed in ref. [28]. Experimental NMR study of various conformations of alanine heptapeptides at different temperatures was carried out in ref. 29]. In ref. 30] with the use of empirical molecular dynamics based on Monte-Carlo methods, a polypeptide consisting of 21 amino acids was described.

In the present paper we have performed an $a b$ initio calculation of the multidimensional potential energy surface for the glycine polypeptide chains consisting of three and six amino acids. The potential energy surface versus twisting degrees of freedom of the polypeptide chain has been calculated. The calculations have been performed within ab initio theoretical framework based on the density functional theory (DFT) accounting for all the electrons in the system. Previously, this kind of calculations were performed only for dipeptides 
(see, e.g., [11, 12, 19]). For larger molecules, only a few conformations were considered (see citations above). We have calculated the energy barriers for the transitions between different molecular conformations and determined the energetically most favorable ones. Using a thermodynamic approach, we have estimated times of the characteristic transitions between the most energetically favorable conformations. The results of our calculation have been compared with other theoretical simulations and with the available experimental data. We have also analysed how the secondary structure of polypeptide chains influences the potential energy landscapes. In particular, the role of the secondary structure in the

formation of stable conformations of the chains of six amino acids being in the sheet and in the helix conformations has been elucidated. Some preliminary results of our work were published as electronic preprints [31, 32].

Our paper is organized as follows. In section [I] we give a brief overview of theoretical methods used in our work. In section III we present and discuss the results of our computations. In section [V we draw a conclusion to this paper. The atomic system of units, $|e|=m_{e}=\hbar=1$, is used throughout the paper unless other units are indicated.

\section{THEORETICAL METHODS}

In the present paper we study the multidimensional potential energy surfaces for glycine polypeptides within the framework of the density functional theory. The potential energy surfaces are multidimensional functions of atomic coordinates. In our work the potential energy surfaces were considered as a function of the dihedral angles formed by the atoms of the polypeptide chain. For this calculation the Born-Oppenheimer approximation allowing to separate the motion of the electronic and ionic subsystems is used.

The density functional theory (DFT) is a common tool for the calculation of properties of quantum many body systems in which many electron correlations play an important role. The DFT formalism is well known and can be found in many textbooks (see e.g. [33, 34]). Therefore in our work we present only the basic equations and ideas of this method.

Electronic wave functions and energy levels within the framework of DFT are obtained from the Kohn-Sham equations, which read as (see e.g. 33, 34]):

$$
\left(\frac{\hat{p}^{2}}{2}+U_{i o n s}+V_{H}+V_{x c}\right) \psi_{i}=\varepsilon_{i} \psi_{i}, \quad i=1 \ldots N
$$


where the first term represents the kinetic energy of the $i$-th electron with the wavefunction $\psi_{i}$ and the energy $\varepsilon_{i}, U_{\text {ions }}$ describes the electron attraction to the ionic centers, $V_{H}$ is the Hartree part of the interelectronic interaction [35], $V_{x c}$ is the local exchange-correlation potential.

The exchange-correlation potential is defined as a functional derivative of the exchangecorrelation energy functional:

$$
V_{x c}=\frac{\delta E_{x c}[\rho]}{\delta \rho(\vec{r})}
$$

Equation (21) is exact and follows from the Hohenberg theory [36]. However, no unique potential $E_{x c}$, universally applicable for different systems and conditions, has been found so far.

Approximate functionals employed by the DFT usually partition the exchange-correlation energy into two parts, referred to as the exchange and the correlation terms:

$$
E_{x c}[\rho]=E_{x}(\rho)+E_{c}(\rho)
$$

Both terms are the functionals of the electron density, which can be of two distinctly different types: either a local functional depending only on the electron density $\rho$ or a gradientcorrected functionals depending on both $\rho$ and its gradient, $\nabla \rho$. A variety of exchange correlation functionals can be found in literature. In our work we have used the hybrid Becke-type three-parameter exchange functional 37] paired with the gradient-corrected Lee, Yang and Parr correlation functional $(B 3 L Y P)$ [38, 39].

\section{RESULTS AND DISCUSSION}

\section{A. Determination of the polypeptide twisting degrees of freedom}

In this section we present the potential energy surfaces for the glycine polypeptide chains calculated versus dihedral angles $\varphi$ and $\psi$ defined in figure 1. In particular, we focus on the chains consisting of three and six amino acids.

Both angles are defined by the four neighboring atoms in the polypeptide chain. The angle $\varphi_{i}$ is defined as the dihedral angle between the planes formed by the atoms $\left(C_{i-1}^{\prime}-N_{i}-C_{i}^{\alpha}\right)$ and $\left(N_{i}-C_{i}^{\alpha}-C_{i}^{\prime}\right)$. The angle $\psi_{i}$ is defined as the dihedral angle between $\left(N_{i}-C_{i}^{\alpha}-C_{i}^{\prime}\right)$ and 


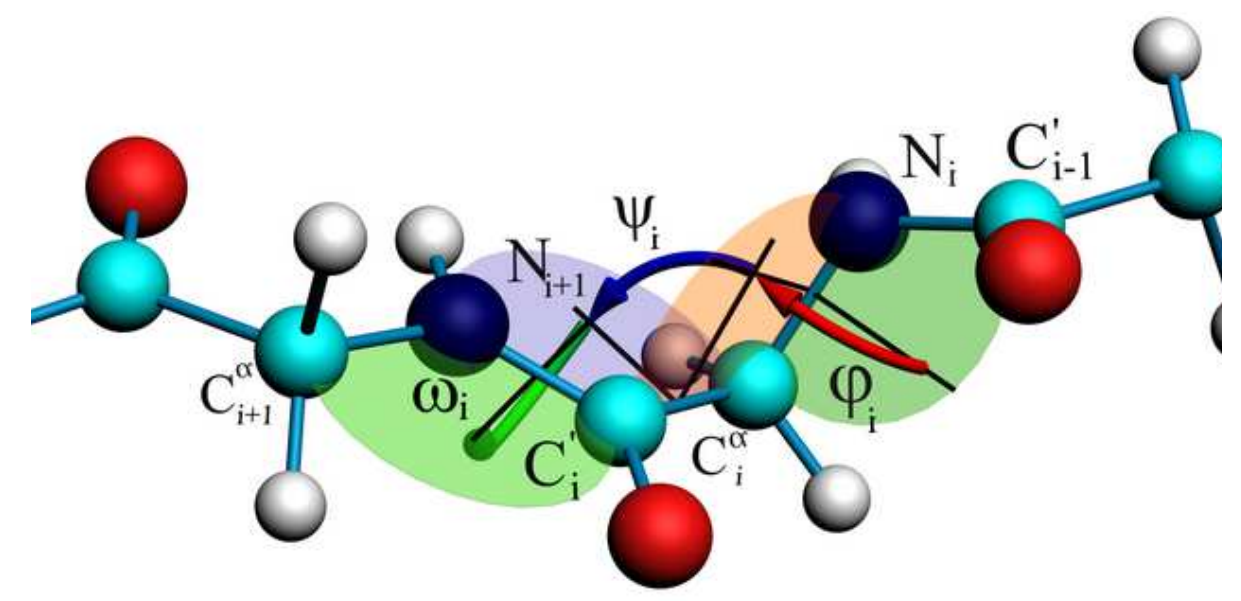

Hydrogen

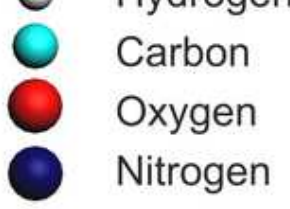

FIG. 1: Dihedral angles $\varphi$ and $\psi$ used to characterize the potential energy surface of the polypeptide chain.

$\left(C_{i}^{\alpha}-C_{i}^{\prime}-N_{i+1}\right)$ planes. Beside the angles $\varphi_{i}$ and $\psi_{i}$ there is an angle $\omega_{i}$, which is defined as the dihedral angle between the $\left(C_{i}^{\alpha}-C_{i}^{\prime}-N_{i+1}\right)$ and $\left(C_{i}^{\prime}-N_{i+1}-C_{i+1}^{\alpha}\right)$ planes. The atoms are numbered from the $\mathrm{NH}_{2}$ - terminal of the polypeptide. The angles $\varphi_{i}, \psi_{i}$ and $\omega_{i}$ take all possible values within the interval $\left[-180^{\circ} ; 180^{\circ}\right]$. For the unambiguous definition we count the angles $\varphi_{i}, \psi_{i}$ and $\omega_{i}$ clockwise, if one looks on the molecule from its $\mathrm{NH}_{2}-$ terminal (see fig. 11). This way of angle counting is the most commonly used [10].

The angles $\varphi_{i}$ and $\psi_{i}$ can be defined for any amino acid in the chain except for the first and the last ones. Below we omit the subscripts and consider angles $\varphi$ and $\psi$ for the middle amino acid of the polypeptide.

\section{B. Optimized geometries of glycine polypeptides}

In order to study the twisting of a polypeptide chain one needs first to define its initial structure. Although the number of its conformations increases with the growth of the molecule size, there are certain types of polypeptide structure, namely the sheet and the helix conformations, which are the most typical. In the present paper we have investigated 
twisting of the polypeptide chains of the sheet and the helix conformation. By varying the angles $\varphi$ and $\psi$ in the central amino acid one can create the structure of the polypeptide differing significantly from the pure sheet or helix conformations. If the structure of a polypeptide can be transformed to a helix or a sheet one by a trivial variation of $\varphi$ and $\psi$, such polypeptides are referred below as belonging to the group of the helix or the sheet structure respectively.
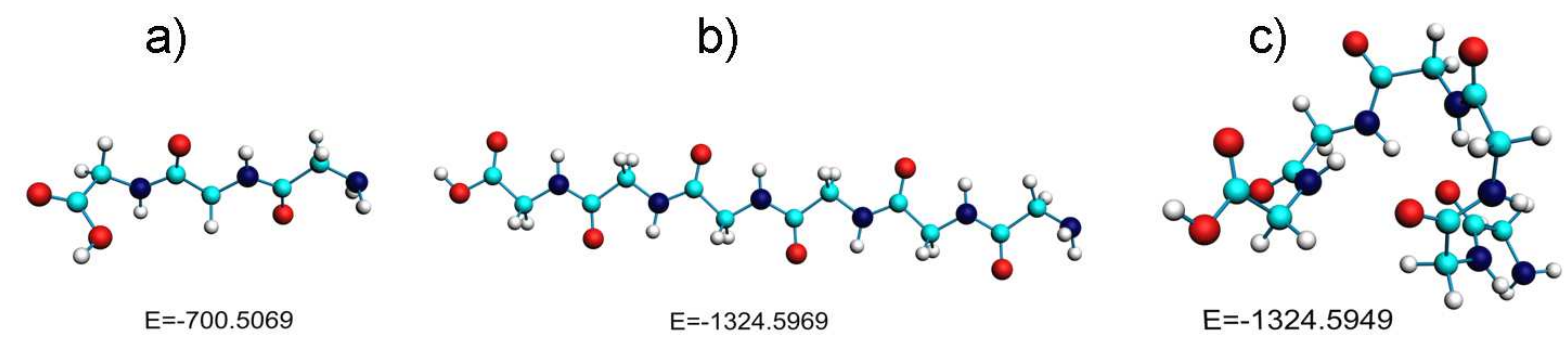

FIG. 2: Optimized geometries of glycine polypeptide chains calculated by the B3LYP/6$31++\mathrm{G}(\mathrm{d}, \mathrm{p})$ method: a) Glycine tripeptide; b) Glycine hexapeptide (sheet conformation); c) Glycine hexapeptide (helix conformation).

In figure 2 we present the optimized geometries of glycine polypeptide chains that have been used for the exploration of the potential energy surfaces. All geometries have been optimized with the use of the B3LYP functional. Figure 2 2 shows the glycine tripeptide structure. In the present work we choose in the sheet conformation, because the tripeptide is too short to form the helix conformation. Figures 2 20 and 2r show glycine hexapeptide in the sheet and the helix conformations respectively. The total energies (in atomic units) of the molecules are given below the images.

\section{Potential energy surface for glycine tripeptide}

In figure 3 we present the potential energy surface for the glycine tripeptide calculated by the B3LYP/6-31G(2d,p) method. The energy scale is given in eV, $\mathrm{kcal} / \mathrm{mol}$ and Kelvin. Energies on the plot are measured from the lowest energy minimum of the potential energy surface.

From the figure follows that there are several minima on the potential energy surface. They are numbered according to the value of the corresponding energy value. Each minimum corresponds to a certain conformation of the molecule. These conformations differ 


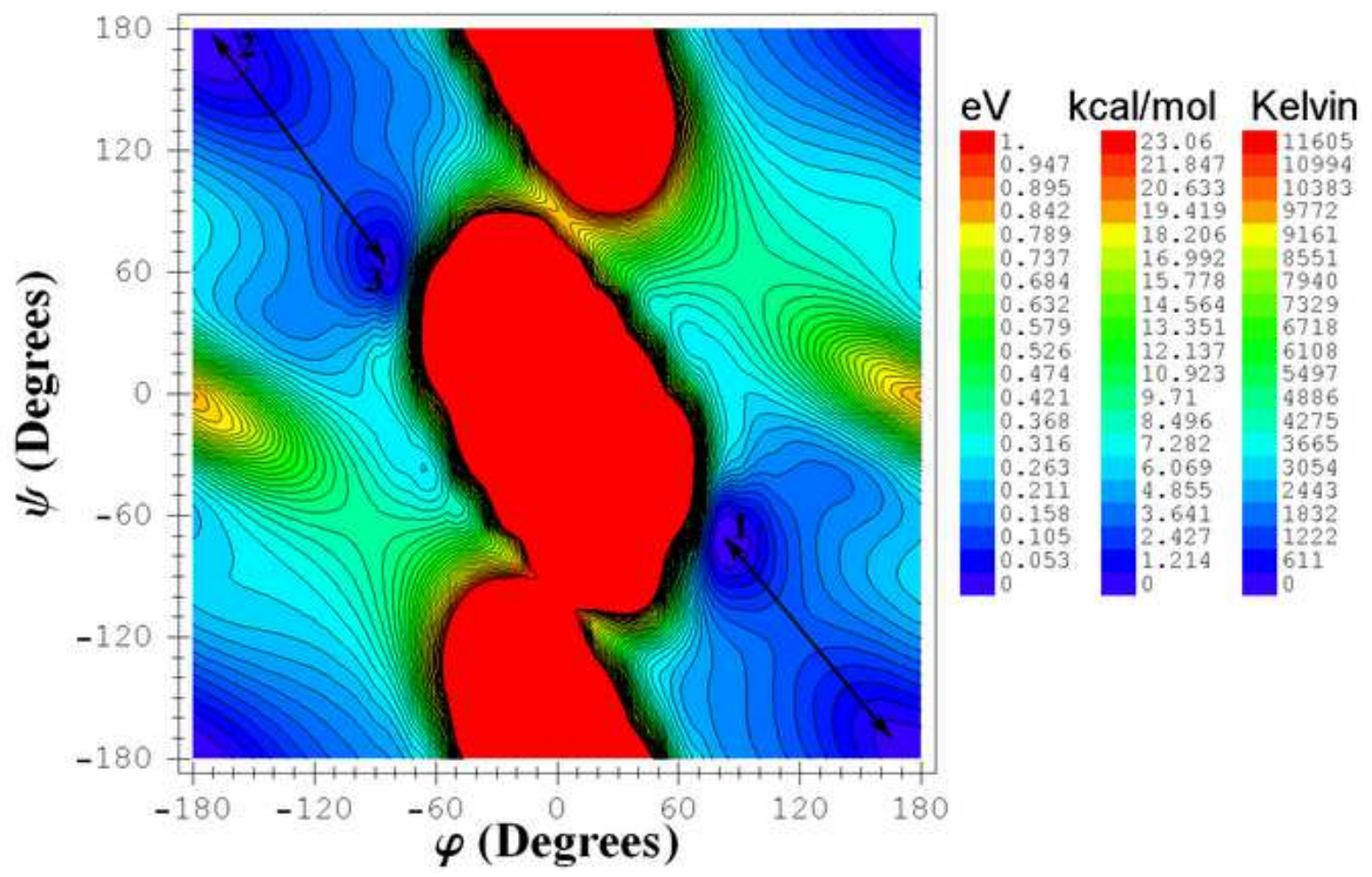

FIG. 3: Potential energy surface for the glycine tripeptide calculated by the B3LYP/6-31G(2d,p) method. Energies are given in $\mathrm{eV}, \mathrm{kcal} / \mathrm{mol}$ and Kelvin. Numbers mark energy minima on the potential energy surface. Arrows show the transition paths between different conformations of the molecule.

significantly from each other. In the case of glycine tripeptide there are only three conformations, shown in figure 4. Dashed lines show the strongest hydrogen bonds in the system, which arise when the distance between hydrogen and oxygen atoms becomes less then 2.9 angstroms.

To calculate the potential energy surface the following procedure was adopted. Once the stable structure of the molecule has been determined and optimized, all but two (these are the angles $\varphi$ and $\psi$ in the central amino acid) degrees of freedom were frozen. Then the energy of the molecule was calculated by varying $\varphi$ and $\psi$. This procedure was used to calculate all potential energy surfaces presented below in this section. It allows one to find efficiently the minima on the potential energy surface and to determine the main stable conformations of the molecule. The absolute energy values of different conformations of the tripeptide found by this method are not too accurate, because the method does not account for the relaxation of other degrees of freedom in the system. To calculate the 


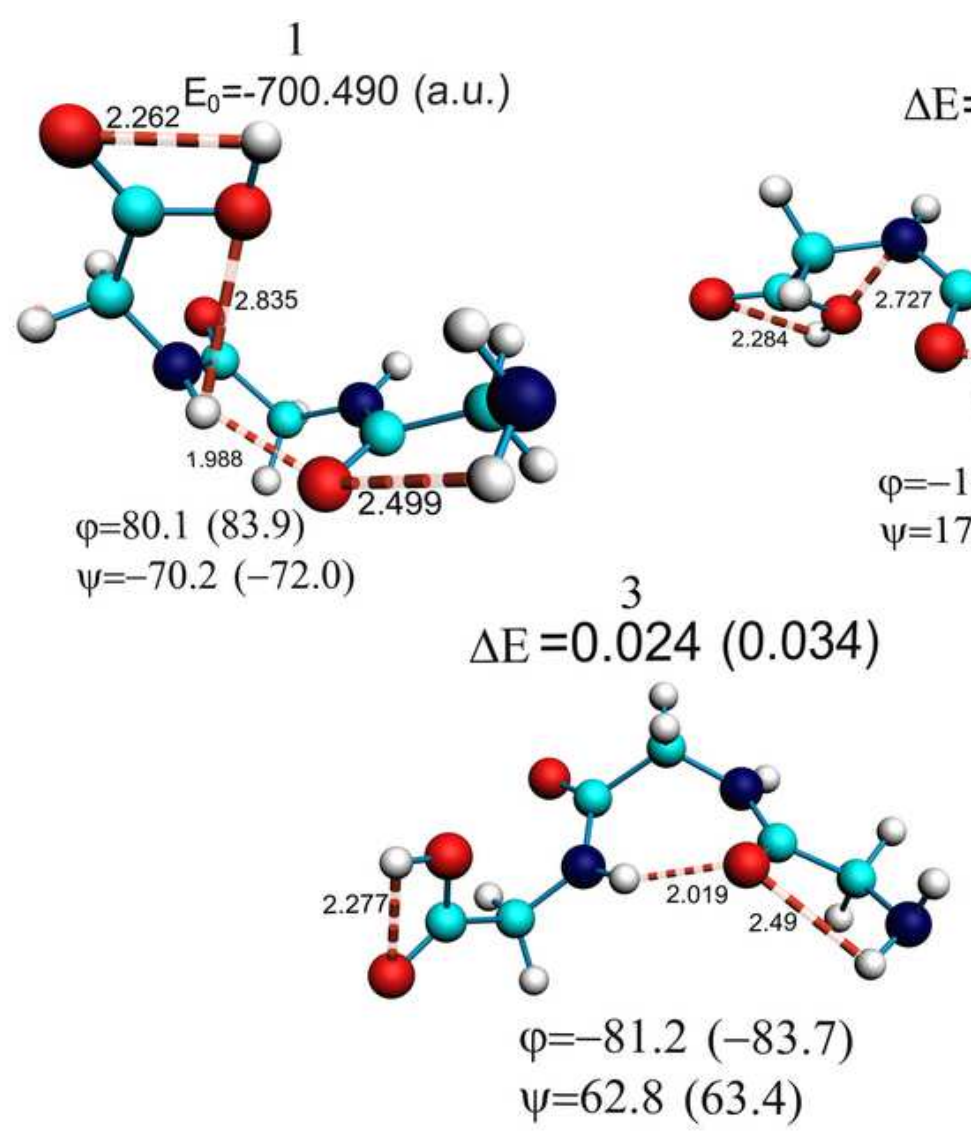

FIG. 4: Optimized conformations of the glycine tripeptide. Different geometries correspond to different minima on the potential energy surface (see contour plot in figure 3). Below each image we present angles $\varphi$ and $\psi$, which have been obtained with accounting for relaxation of all degrees of freedom in the system. Values in brackets give the angles calculated without accounting for relaxation. Above each image, the energy of the corresponding conformation is given in $\mathrm{eV}$. The energies are counted from the energy of conformation 1 (the energy of conformation 1 is given in a.u.). Values in brackets give the energies obtained without accounting for the relaxation of all degrees of freedom in the system. Dashed lines show the strongest hydrogen bonds. Their lengths are given in angstroms.

potential energy surface with accounting for the relaxation one needs 20-30 times more of the computer time. Therefore, a systematic calculations with accounting for the relaxation have not been performed in our work. Instead, we have performed a complete optimization of the molecular conformations, corresponding to all minima on the calculated potential energy surface. 
In figure 4 we compare stable conformations of the glycine tripeptide calculated with and without accounting for the relaxation of all atoms in the system. As it is seen from this figure the angles $\varphi$ and $\psi$ differ by about 10 percent in the two cases. This difference arises due to the coupling of $\varphi$ and $\psi$ with other degrees of freedom. Note the change of the sign of the relative energies of some conformations. This effect is due to the rearrangement of side atoms (radicals) in the polypeptide chain which lowers the energies of different conformations differently.

In our work the potential energy surface has been calculated and interpolated on the grid with the step of $18^{\circ}$. This step size is an optimal one, because the interpolation error is about $9^{\circ}$, i.e. comparable with the angle deviations caused by the relaxation of all degrees of freedom in the system.

TABLE I: Comparison of dihedral angles $\varphi$ and $\psi$ corresponding to different conformations of glycine tripeptide (column 3) with angles $\varphi$ and $\psi$ for glycine dipeptide from 11, 12] (column 1 and 2).

\begin{tabular}{c|cc|cc|cc}
\hline \hline conformation & $\varphi,[11]$ & $\psi[11]$ & $\varphi[12]$ & $\psi[12]$ & $\varphi$ & $\psi$ \\
\hline 1 & - & - & 76.0 & -55.4 & 80.1 & -70.2 \\
2 & -180.0 & 180.0 & -157.2 & 159.8 & -164.2 & 176.2 \\
3 & -85.2 & 67.4 & -85.8 & 79.0 & -81.2 & 62.8 \\
\hline \hline
\end{tabular}

In refs. [11] and [12] several stable conformations were found for alanine and glycine dipeptides. The values of angles $\varphi$ and $\psi$ for the stable conformations of dipeptide and tripeptide are close indicating that the third amino acid in tripeptide makes relatively small influence on the values of dihedral angles of two other amino acids. In earlier papers [11, 12] dipeptides were studied within the framework of the Hartree-Fock theory. In ref. [11], values of $\varphi$ and $\psi$ were obtained by the $\mathrm{HF} / 6-31+\mathrm{G}^{*}$ method, and in ref. [12] by $\mathrm{HF} / 6-31 \mathrm{G}^{* *}$. In table \we compare the results of our calculation for tripeptide with the corresponding data obtained for dipeptides. Some discrepancy between the values presented is due to the difference between the dipeptide and tripeptide (i.e. the third glycine in tripeptide affects the values of angles $\varphi$ and $\psi$ ). However, another source of discrepancy might arise due to accounting for the many-electron correlations in the DFT and neglecting this effect in the Hartree-Fock theory used in refs. [11, 12]. 


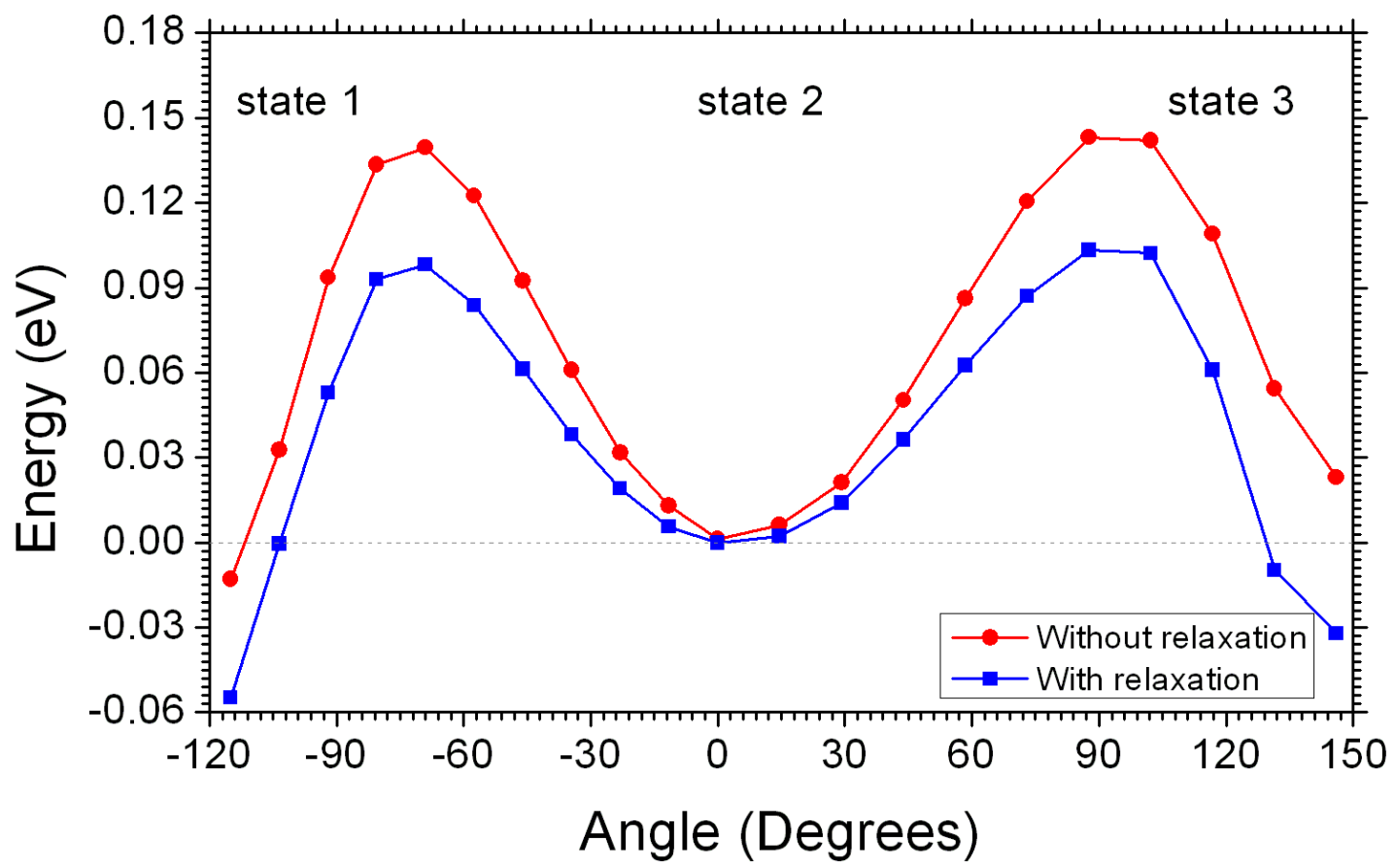

FIG. 5: Transition barriers between conformations $1 \leftrightarrow 2 \leftrightarrow 3$ of the glycine tripeptide. Circles and squares correspond to the barriers calculated without and with relaxation of all degrees of freedom in the system.

Figure [3] shows that some domains of the potential energy surface, where the potential energy of the molecule increases significantly, appear to be unfavorable for the formation of a stable molecular configuration. The growth of energy takes place when some atoms in the polypeptide chain approach each other of small distances. Accounting for the molecule relaxation results in the decrease of the system energy in such cases, but the resulting molecular configurations remain unstable. We call such domains on the potential energy surface as forbidden ones. In figure 3 one can identify two forbidden regions in the vicinity of the points $(0,0)$ and $(0,180)$. At $(0,0)$ a pair of hydrogen and oxygen atoms approach to the distances much smaller than the characteristic $H-O$ bond length. This leads to a strong interatomic repulsion caused by the exchange interaction of electrons. At $(0,180)$ the Coulomb repulsion of pair of oxygen atoms causes the similar effect.

Figure 3 shows that there are three minima on the potential energy surface for glycine tripeptide. The transition barriers between the conformations $2 \leftrightarrow 1$ and $2 \leftrightarrow 3$ are shown in figure 5. They have been calculated with and without relaxation of the atoms in the system. The corresponding transition paths are marked in figure [3 by arrows. This comparison 
demonstrates that accounting for the relaxation significantly lowers the barrier height and influences the relative value of energy of the minima.

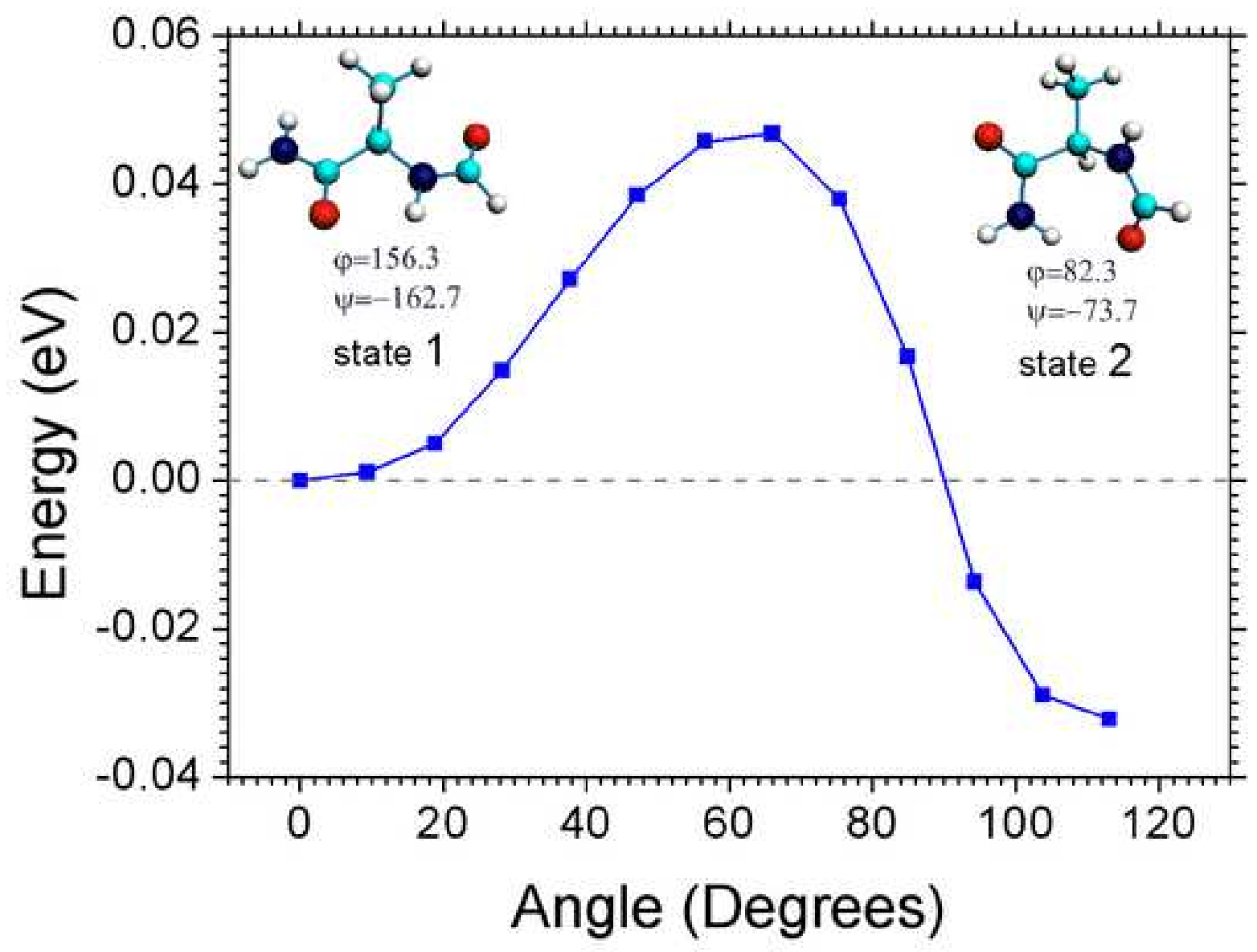

FIG. 6: Transition barriers between conformations $1 \leftrightarrow 2$ of alanine dipeptide analog calculated by the B3LYP $/ 6-31+\mathrm{G}(2 \mathrm{~d}, \mathrm{p})$ method accounting for the relaxation of all degrees of freedom in the system. Structure of the conformations 1 and 2 is shown near each minimum.

Let us now estimate the time needed for a system for the transition from one conformation to another. To do this we use the Arhenius equation, which reads as:

$$
\frac{1}{\tau}=\Omega e^{-\frac{\Delta E}{k T}}
$$

where $\tau$ is the transition time, $\Omega$ is the factor, determining how frequently the system approaches the barrier, $\Delta E$ is the barrier height, $T$ is the temperature of the system, $k$ is the Bolzmann factor.

Figure 6] shows the barrier for the transition between the two main conformations of the 
alanine dipeptide analog ((S)- $\alpha$-(formylamino)propanamide). It is seen that $\Delta E_{1 \rightarrow 2}=0.047$ $\mathrm{eV}$ for the transition $1 \rightarrow 2$, while $\Delta E_{2 \rightarrow 1}=0.079 \mathrm{eV}$ for the transition $2 \rightarrow 1$. The frequency $\Omega$ for this molecule is equal to $42.87 \mathrm{~cm}^{-1}$. Thus, at $T=300 \mathrm{~K}$, we obtain $\tau_{2 \times A l a}^{1 \rightarrow 2} \sim 5$ ps and $\tau_{2 \times A l a}^{2 \rightarrow 1} \sim 17 \mathrm{ps}$. This result is in excellent agreement with the molecular dynamics simulations results obtained in [19] predicting $\tau \sim 7 \mathrm{ps}$ for the transition $1 \rightarrow 2$ and $\tau \sim 19$ ps for the transition $2 \rightarrow 1$. This comparison demonstrates that our method is reliable enough and it can be used for the estimation of transition times between various conformations of the polypeptides.

Using the B3LYP/6-31G(2d,p) method we have calculated the frequencies of normal vibration modes for the glycine tripeptide. The characteristic frequency corresponding to twisting of the polypeptide chain is equal to $33.49 \mathrm{~cm}^{-1}$. From figure 5 follows that $\Delta E_{2 \rightarrow 3}=$ $0.103 \mathrm{eV}$ for the transition $2 \rightarrow 3$, and $\Delta E_{3 \rightarrow 2}=0.132 \mathrm{eV}$ for the transition $3 \rightarrow 2$. Thus, we obtain $\tau_{3 \times G l y}^{2 \rightarrow 3} \sim 54$ ps and $\tau_{3 \times G l y}^{3 \rightarrow 2} \sim 164$ ps. Let us note that these transition times can be measured experimentally by means of NMR 10,40$]$.

\section{Potential energy surface for glycine hexapeptide with the sheet and the helix secondary structure}

In figure 7 we present contour plots of the potential energy surfaces for the glycine hexapeptide with the sheet (part a) and the helix (part b) secondary structure, respectively, versus dihedral angles $\varphi$ and $\psi$. In both cases the forbidden regions arise because of the repulsion of oxygen and hydrogen atoms analogously to the glycine tripeptide case.

Minima 1-5 on the potential energy surface 7 h correspond to different conformations of the glycine hexapeptide with the sheet secondary structure. Note that minima 1-3 are also present on the potential energy surface of the glycine tripeptide. Geometries of the conformations 1-5 are shown on the right-hand side of figure $7 \mathrm{~h}$.

For the glycine hexapeptide with the sheet secondary structure additional minima 4-5 arise. The appearance of these minima is the result of the interaction of the outermost amino acids, which are absent in the case of tripeptide.

Energy barrier as a function of a scan variable (see figure $7 \mathrm{a}$ ) for the transition between conformations 1 and 2 is shown in figure 8 . The energy dependence has been calculated with and without relaxation of all the atoms in the system. In the case of glycine hexapeptide 

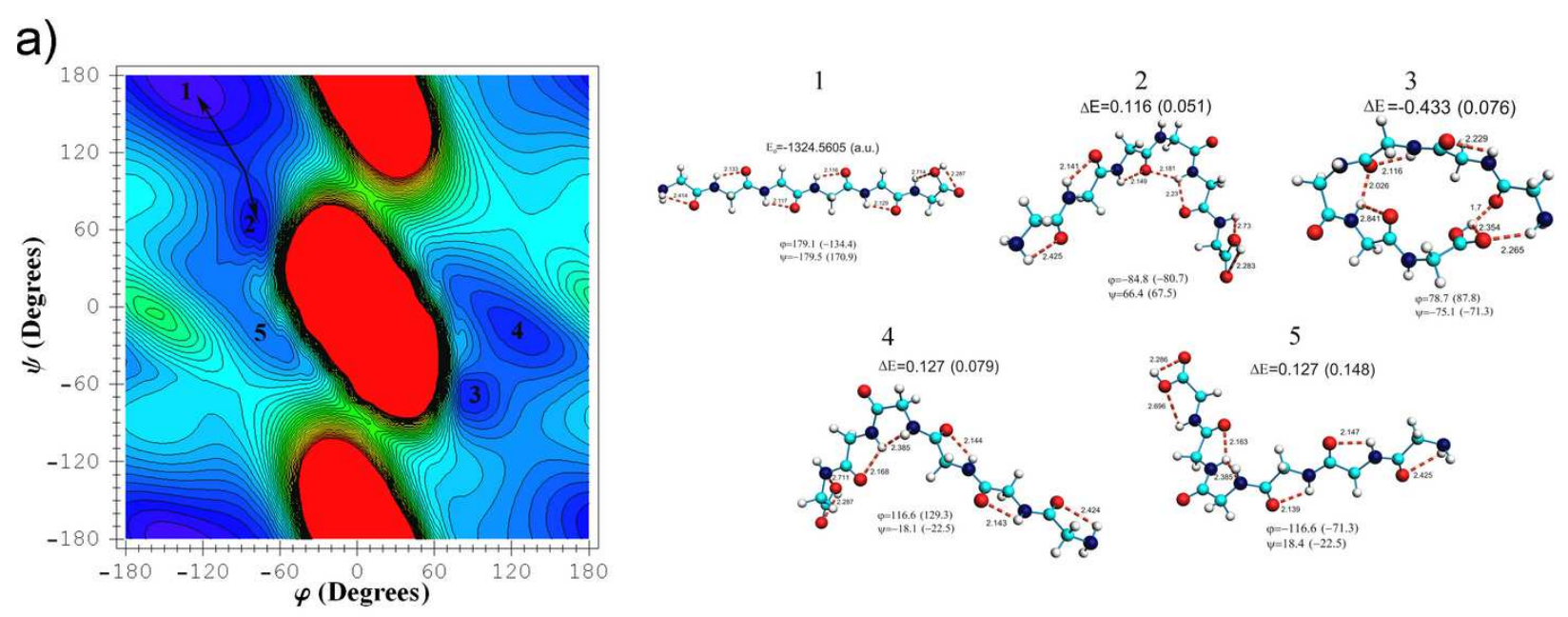

b)
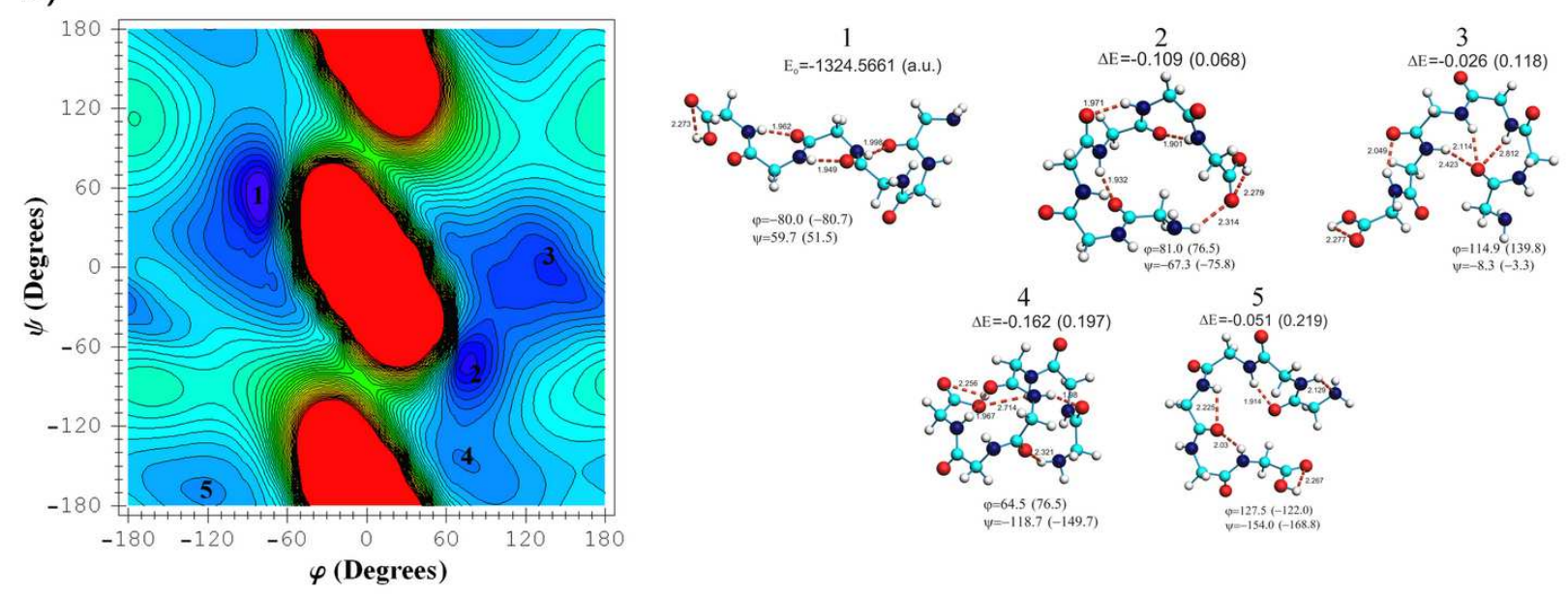

FIG. 7: Potential energy surface for the glycine hexapeptide with the sheet secondary structure (part a) and with the helix secondary structure (part b) calculated by the B3LYP/6-31G(2d,p) method. Energy scale is given in figure 3. Numbers mark energy minima on the potential energy surface. Images of optimized conformations of the glycine hexapeptide are shown near the corresponding energy landscape. Values of angles $\varphi$ and $\psi$, as well as the relative energies of the conformations are given analogously to that in figure 4 .

with the sheet secondary structure the barrier height $(0.128 \mathrm{eV})$ for the transition $1 \rightarrow 2$ appears to be close to the corresponding barrier height of the glycine tripeptide $(0.103 \mathrm{eV})$, while the barrier height for the transition $2 \rightarrow 1$ is significantly lower $(0.028 \mathrm{eV})$. The normal vibration mode frequency, corresponding to the twisting of the polypeptide chain is equal to $15.45 \mathrm{~cm}^{-1}$ and was calculated with the B3LYP/6-31G(2d,p) method. Using equation (4) 


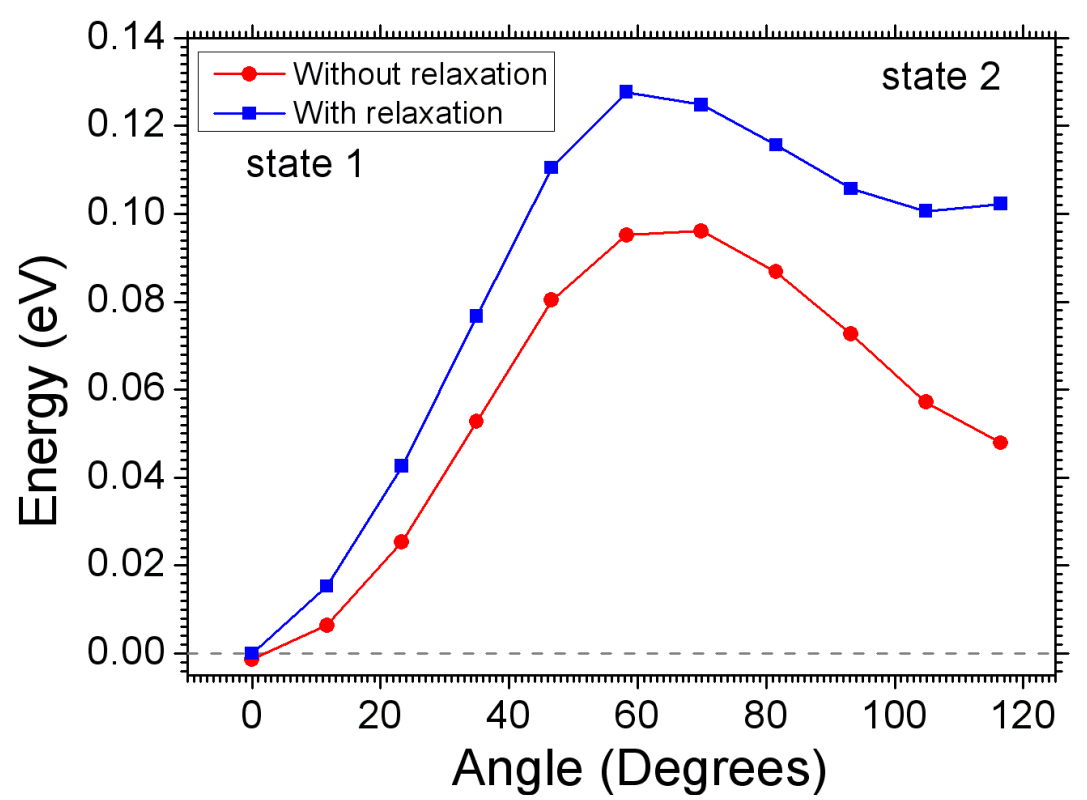

FIG. 8: Transition barriers between conformations $1 \leftrightarrow 2$ of the glycine hexapeptide with the sheet secondary structure. Circles and squares correspond to the barriers calculated without and with relaxation of all degrees of freedom in the system.

one derives the transition times at room temperature: $\tau_{6 \times G l y}^{1 \rightarrow 2} \sim 305 \mathrm{ps}, \tau_{6 \times G l y}^{2 \rightarrow 1} \sim 6 \mathrm{ps}$.

Let us now consider glycine hexapeptide with the helix secondary structure. The potential energy surface for this polypeptide is shown in figure $7 \mathrm{~b}$. The positions of minima on this surface are shifted significantly compared to the cases discussed above. This change takes place because of the influence of the secondary structure of the polypeptide on the potential energy surface. The geometries of the most stable conformations are shown on the right hand-side of figure $7 \mathrm{~b}$.

It is worth noting that for some conformations of glycine hexapeptide the angles $\varphi$ and $\psi$ change significantly when the relaxation of all degrees of freedom in the system is accounted for (see for example conformations 1, 4, 5 in fig. 77 and conformations 3, 4 in fig. [7b). This means that the potential energy surface of the glycine hexapeptide in the vicinity of mentioned minima is very sensitive to the relaxation of all degrees of freedom. However, calculation of the potential energy surface with accounting for the relaxation of all degrees of freedom is unfeasible task. Indeed, one needs about 1000 hours of computer time (Pentium Xeon 2.4 GHz) for the calculation of the potential energy surface for the glycine hexapeptide. To perform an analogues calculation with accounting for the relaxation about 
3 years of computer time would be needed. Nevertheless, the potential energy surface calculated without accounting for the relaxation carries a lot of useful information. Thus, one can predetermine stable conformations of polypeptide, which then can be used as starting configurations for further energy minimization.

\section{E. Comparison of calculation results with experimental data}

Nowadays, the structure of many proteins has been determined experimentally [9]. Knowing the protein structure one can find the angles $\varphi$ and $\psi$ for each amino acid in the protein.

In figure 97, we show a map of the allowed and forbidden conformations for glycine residues in poly-glycine chain taken from [42] (steric Ramachandran diagram). This map was obtained from pure geometrical considerations, in which the structure of the polypeptide was assumed to be fixed and defined by the interatomic van der Waals interaction radii. Depending on the distances between the atoms one could distinguish three regions: completely allowed, conventionally allowed and forbidden. The conformation is called completely allowed if all the distances between atoms of different amino acids are larger than some critical value $r_{i j} \geq r_{\max }$. Conventionally allowed regions on the potential energy surface correspond to the conformations of the polypeptide, in which the distances between some atoms of different amino acids lie within the interval $r_{\min } \leq r_{i j}<r_{\max }$. All other conformations are referred to as forbidden. The values of $r_{\min }$ and $r_{\max }$ are defined by the types of interacting atoms and can be found in the textbooks (see, e.g., [42]). In figure 9 a we mark the completely allowed regions with white, the conventionally allowed regions with light-gray and the forbidden regions with dark gray color. In this figure we mark the points, which correspond to the geometries of glycine, whose periodical iteration leads to the formation of chains with specific secondary structure. In table II we compile the values of angles $\varphi$ and $\psi$, which correspond to the most prominent poly-glycine secondary structures. For the illustrative purposes we mark these points by white circles with the corresponding type of the secondary structure typed in. Thus, $2_{7}^{R}, 2_{7}^{L}$ are the right-handed and the left-handed $2_{7}$ helix; $3_{10}^{R}, 3_{10}^{L}$ are the right-handed and the left-handed $3_{10}$ helix; $\alpha_{R}, \alpha_{L}$ are the righthanded and the left-handed $\alpha$-helix $\left(4_{13}\right) ; \pi_{R}, \pi_{L}$ are the right-handed and the left-handed $\pi$-helix $\left(5_{16}\right) ; \uparrow \uparrow, \uparrow \downarrow$ are the parallel and antiparallel $\beta$ sheets. $\beta_{I}, \beta_{I I}$ correspond to the $\beta$-turns of types I and II respectively. 
a)

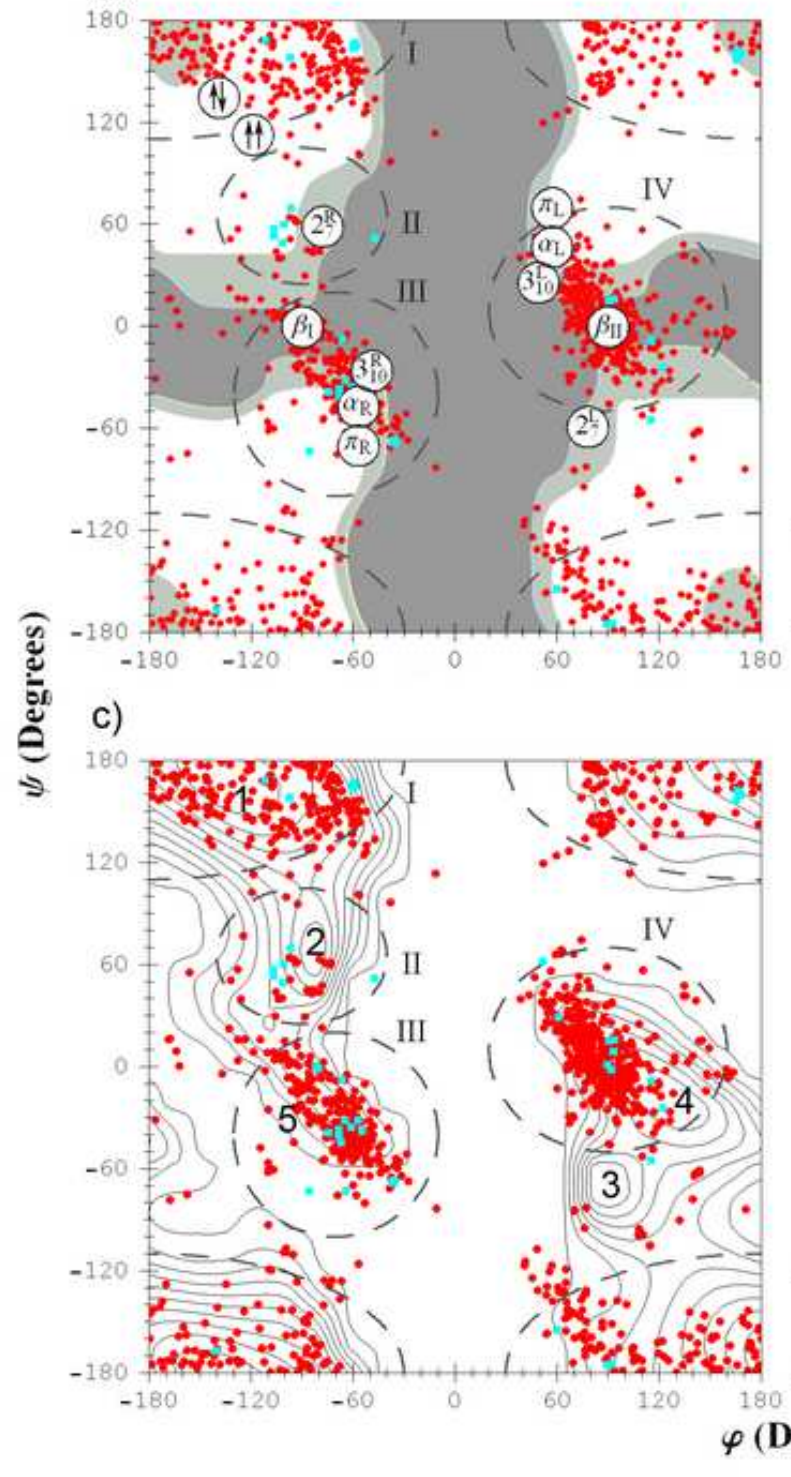

b)

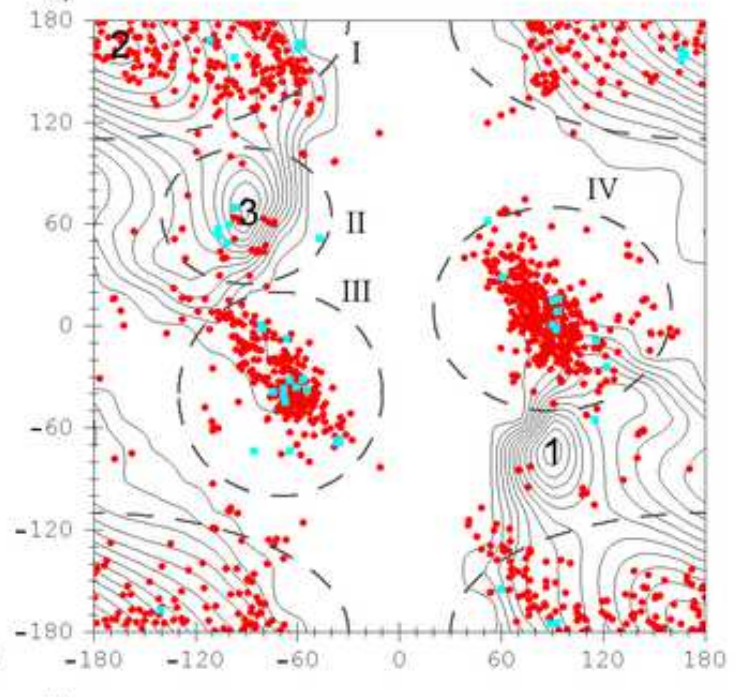

d)

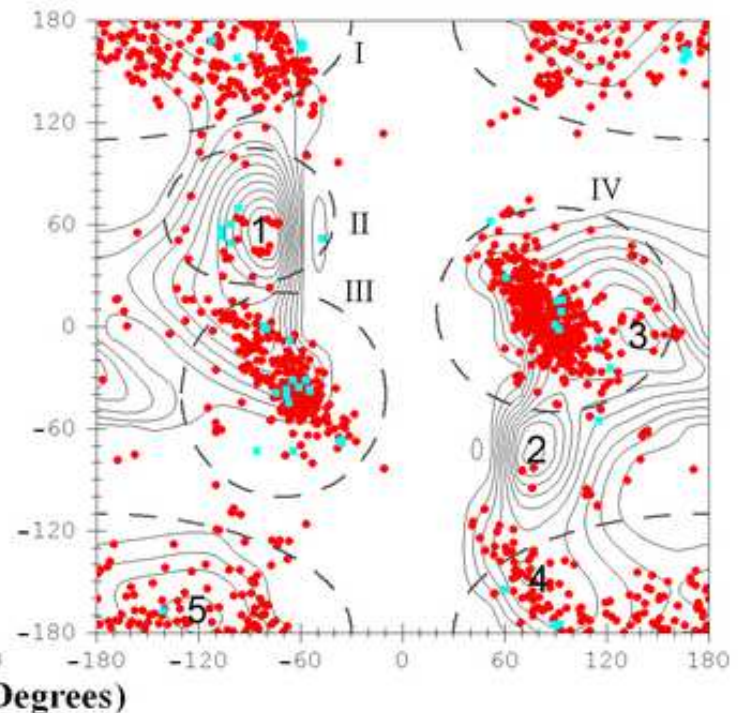

FIG. 9: Comparison of angles $\varphi$ and $\psi$ of glycine residues in protein structures selected from the Brookhaven Protein Data Bank 9, 41] with the steric diagram for poly-glycine [42] (part a)). Comparison of angles $\varphi$ and $\psi$ of glycine residues in protein structures selected from the Brookhaven Protein Data Bank 9, 41] with the minima on the calculated potential energy surfaces for: glycine tripeptide (b); glycine hexapeptide in sheet conformation (c); glycine hexapeptide in helix conformation (d). Transparent rhomboids correspond to glycines surrounded with glycines, while filled circles correspond to glycines surrounded by other amino acids. Dashed ellipses mark the regions of higher concentration of the observed angles. 
TABLE II: Angles $\varphi$ and $\psi$ corresponding to the most prominent poly-glycine secondary structures.

\begin{tabular}{ccc}
\hline \hline Structure type & $\varphi$ (Deg.) & $\psi$ (Deg.) \\
\hline right-handed (left-handed) $2_{7}$ helix & $-78(78)$ & $59(-59)$ \\
right-handed (left-handed) $3_{10}$ helix & $-49(49)$ & $-26(26)$ \\
right-handed (left-handed) $\alpha-$ helix $\left(4_{13}\right)$ & $-57(57)$ & $-47(47)$ \\
right-handed (left-handed) $\pi-$ helix $\left(5_{16}\right)$ & $-57(57)$ & $-70(70)$ \\
parallel $\beta$ sheet $(\uparrow \uparrow)$ & -119 & 113 \\
antiparallel $\beta$ sheet $(\uparrow \downarrow)$ & -139 & 135 \\
$\beta$-turn of type I & -90 & 0 \\
$\beta$-turn of type II & 90 & 0 \\
\hline \hline
\end{tabular}

Note that not all of the structures listed above are present equally in proteins. In figure 9a we show the distribution of the angles $\varphi$ and $\psi$ of glycine residues in protein structures selected from the Brookhaven Protein Data Bank 9, 41]. It is possible to distinguish four main regions, in which most of the experimental points are located. In figure 9 these regions are schematically shown with dashed ellipses. Note that these ellipses are used for illustrative purposes only, and serve for a better understanding of the experimental data. The regions in which most of the observed angles $\varphi$ and $\psi$ are located correspond to different secondary structure of the poly-glycine. Thus, region I corresponds to the parallel and antiparallel $\beta$-sheets. Region II corresponds to the right-handed $2_{7}^{R}$ helix. Region III corresponds to the right-handed $\alpha_{R}$-helix, right-handed $3_{10}^{R}$ helix, right-handed $\pi_{R}$-helix and $\beta$-turn of type I. Region IV corresponds to the left-handed $\alpha_{L}$-helix, left-handed $3_{10}^{L}$ helix and $\beta$-turn of type II. In some cases there are several types of secondary structure within one domain. In the present work we have not studied the secondary structure of proteins systematically enough to establish the univocal correspondence of the observed experimental points to different types of the secondary structure. Figure 9a demonstrates that $\pi_{L}$ and $2_{7}^{L}$ helixes are rarely observed in experiment.

Note that some experimental points lie in the forbidden region of the steric Ramachandran diagram (see region IV in fig. 9a). The quantum calculation shows that in fact this region is allowed and has several minima on the potential energy surface (see fig 9k and 9d). This comparison shows that for an accurate description of polypeptides it is important to take 
quantum properties of these systems into account.

Let us now compare the distribution of angles $\varphi$ and $\psi$ experimentally observed for proteins with the potential energy landscape calculated for glycine polypeptides, and establish correspondence of the secondary structure of the calculated conformations with the predictions of the simple Ramachandran model.

Region I corresponds to the minima 2, 1 and 5 on the potential energy surfaces of the glycine tripeptide (fig. 9b), the glycine hexpeptide with the secondary structure of sheet (fig. 9r), and the glycine hexapeptide with the secondary structure of helix (fig. 9d) respectively. Conformations 2 and 1 in figures 9b and 9c correspond exactly to the glycine chains in the $\beta$-sheet conformation (see fig. 4 and $7 \mathrm{a}$ ). Conformation 5 in figure 9d is a mixed state. Here the central amino acid has the conformation of sheet, while the outermost amino acids have the conformation of helix.

Region II corresponds to the minima 3, 2 and 1 on the potential energy surfaces 9], 9k, and 9d, respectively. On the steric diagram for poly-glycine (see fig. 9a) this region corresponds to the right-handed $2_{7}^{R}$ helix. The structure of conformations 3 and 2 on the surfaces 9b and 9c differs from the structure of this particular helix type. Only the central glycines, for which the angles $\varphi$ and $\psi$ in figures 9b and 9re are defined, have the structure of $2_{7}^{R}$ helix. Thus, one can refer to conformations 3 and 2 as to the mixed states, where the central part of the polypeptide chain has the conformation of helix and the outermost parts have the conformation of sheet. Conformation 1 on the surface $9 \mathrm{~d}$ is also a mixed state. Here one can distinguish one turn of $3_{10}^{R}$ helix and two turns of $2_{7}^{R}$ helix (see fig. [7b).

Region III corresponds to the structure of right-handed $\alpha_{R}$-helix, right-handed $3_{10}^{R}$ helix, right-handed $\pi_{R}$-helix and $\beta$-turn I. It corresponds to minimum 5 on the potential energy surface of the glycine hexapeptide with the secondary structure of sheet 9 . Conformation 5 can be characterized as partially formed $\beta$-turn, because the glycine, for which the dihedral angles $\varphi$ and $\psi$ in figure (fig. 9r) are defined, has the geometry of $\beta$-turn and its neighbor forms a $\beta$-sheet (see fig. [7a). There are no minima in region III on the potential energy surfaces presented in figures 9b and 9d. This happens because in this case most probable is the structure of right-handed $\alpha_{R}$-helix. To form one turn of the helix of this type it is necessary to link at least four amino acids, so the glycine tripeptide is too short for that. As well, six amino acids chain is too short to form a stable fragment of an $\alpha_{R}$-helix, because it does not have enough hydrogen bonds to stabilize the structure. For the hexapeptide more 
probable are the elements of $3_{10}^{R}$ and $2_{7}^{R}$ helixes, because in these cases 2 and 3 helical turns respectively can be formed.

Region IV is represented by the structure of left-handed $\alpha_{L}$-helix, left-handed $3_{10}^{L}$ helix and $\beta$-turn of type II. To form these structures it is necessary to have at least four amino acids, therefore there is no minima in this region on the potential energy surface of glycine tripeptide (fig. 9]). Region IV corresponds to the conformations 4 and 3 on the surfaces 9r and 9d respectively. Conformation 4 on the surface 9c corresponds to partially formed $\beta$-turn, because the glycine, for which the dihedral angles $\varphi$ and $\psi$ in figure 9r are plotted has the configuration of $\beta$-turn, but the neighboring aminoacid in the chain forms a $\beta$-sheet (see fig. $7 \mathrm{a}$ ). Conformation 3 on the surface $9 \mathrm{~d}$ can be characterized as deformed turn of left-handed $\alpha_{L}$-helix, which turns out to be energetically the most favorable in this region of the potential energy surface (see fig. 7b).

Finally, we mention a few peculiarities of the calculated potential energy landscapes. At each of the potential energy surfaces discussed in this work one can see a minimum at $\varphi \sim 80^{\circ}$ and $\psi \sim-70^{\circ}$. On the steric diagram for poly-glycine this region corresponds to the left-handed $2_{7}^{L}$ helix. Conformations 1, 3 and 2 on the potential energy surfaces 9p, 9p, and $9 \mathrm{~d}$ respectively partially represent this structure (see fig. 4, 7a and $7 \mathrm{~b}$ ). The structure of conformation 4 on the potential energy surface $9 \mathrm{~d}$ is similar to left-handed $\alpha_{L}$-helix, but differs from it due to the short length of the polypeptide chain, resulting in significant variation of angles $\varphi$ and $\psi$ in all the residues along the chain.

\section{CONCLUSION}

In the present paper the multidimensional potential energy surfaces for amino acid chains consisting of three and six glycines has been investigated and the conformational properties of these systems with respect to the twisting of the polypeptide chain have been described. The calculations were carried out within ab initio theoretical framework based on the density functional theory accounting for all the electrons in the system. We have determined stable conformations and calculated the energy barriers for transitions between them. Using a thermodynamic approach, we have estimated times of the characteristic transitions between the conformations. It was demonstrated that the transition times lie within the picosecond region. Our estimates are compared with the available molecular-dynamics simulations 
results, and the correspondence between the results of the two different methods is reported. A strong barrier asymmetry between neighboring stable conformations on the potential energy surface was found.

We have compared for the first time values of angles $\varphi$ and $\psi$ for glycine residues experimentally observed in real proteins with the coordinates of minima on the potential energy surfaces. This comparison has showed that all profound minima on the potential energy surfaces correspond to the regions in which experimentally measured values of $\varphi$ and $\psi$ are located. We have also analysed how the secondary structure of polypeptide chains influences the formation of the potential energy landscapes. For the chains of six amino acids with the secondary structures of sheet and helix the influence of the secondary structure on the stable conformations of the molecule was demonstrated.

The results of this work can be utilized for modeling more complex molecular systems. For example, the suggested model for the estimation of the characteristic transition times can be used for longer polypeptide chains, also consisting of different amino acids and for estimates of time of proteins folding. It is also possible to use the results of the present work for testing the applicability and accuracy of different model approaches for the polypeptide description requiring much less computer time than ab initio calculations.

\section{ACKNOWLEDGEMENTS}

This work is partially supported by the European Commission within the Network of Excellence project EXCELL, by INTAS under the grant 03-51-6170 and by the Russian Foundation for Basic Research under the grant 06-02-17227-a. We are grateful to Dr. A. Korol and Dr. O. Obolensky for their help in preparation of this manuscript. The possibility to perform complex computer simulations at the Frankfurt Center for Scientific Computing is also gratefully acknowledged.

[1] M. Karas and F. Hillenkamp, Anal. Chem. 60, 2299 (1988).

[2] F. Hillenkamp and M. Karas, Int. J. of Mass Spect. 200, 71 (2000).

[3] M. Karas, U. Bahr, I. Fournier, M. Gluckmann, and A. Pfenninger, J. of Mass Spect. 226, $239(2003)$. 
[4] M. Wind and W. Lehmann, J. Anal. At. Spect. 19, 20 (2004).

[5] J. Fenn, M. Mann, C. Meng, S. Wong, and C. Whitehouse, Science 246, 64 (1989).

[6] S. Bröndsted-Nielsen, J. Andersen, P. Hvelplund, B. Liu, and S. Tomita, J. Phys. B: At. Mol. Opt. Phys. 37, R25 (2004).

[7] A. Finkelstein and O. Ptizin, Physics of Proteins (Moscow University Press "Universitet", 2002).

[8] A. Mülberg, Protein Folding (St. Petersburg University Press, 2004).

[9] H. Berman, J. Westbrook, Z. Feng, G. Gilliland, T. Bhat, H. Weissig, I. Shindyalov, and P. Bourne, Nucleic Acids Research 28, 235 (2000).

[10] A. Rubin, Biophysics: Theoretical Biophysics (Moscow University Press "Nauka", 2004).

[11] T. Head-Gordon, M. Head-Gordon, M. Frisch, C. B. III, and J. Pople, J. Am. Chem. Soc. 113, 5989 (1991).

[12] I. Gould, W. Cornell, and I. Hillier, J. Am. Chem. Soc 116, 9250 (1994).

[13] Z. Wang and Y. Duan, J. Comp. Chem. 25, 1699 (2004).

[14] A. Percel, O. Farkas, I. Jákli, I. Topol, and I. Csizmadia, J. Comp. Chem. 24, 1026 (2003).

[15] I. Húdaky, P. Húdaky, and A. Percel, J. Comp. Chem. 25, 1522 (2004).

[16] R. Improta and V. Barone, J. Comp. Chem. 25, 1333 (2004).

[17] R. Vargas, J. Garza, B. Hay, and D. Dixon, J. Phys. Chem. A 106, 3213 (2002).

[18] R. Kashner and D. Hohl, J. Phys. Chem. A 102, 5111 (1998).

[19] D. Wei, H. Guo, and D. Salahub, Phys. Rev. E 64 (2001).

[20] O. Bludsky̌, J. Chocholoušová, J. Vacek, F. Huisken, and P. Hobza, J. Chem. Phys. 113, 4629 (2000).

[21] S. Woutersen, Y. Mu, G. Stock, and P. Hamm, Chem. Phys. 266 (2001).

[22] S. Woutersen, R. Pfister, Y. Mu, D. Kosov, and G. Stock, J. Chem. Phys. 117, 6833 (2002).

[23] Y. Mu and G. Stock, J. Phys. Chem. B. 106, 5294 (2002).

[24] Y. Mu, D. Kosov, and G. Stock, J. Phys. Chem. B. 107, 5064 (2003).

[25] P. Nguyen and G. Stock, J. Chem. Phys. 119, 11350 (2003).

[26] H. Torii and M. Tasumi, Journ. of Ram. Spect. 29, 81 (1998).

[27] R. Schweitzer-Stenner, F. Eker, Q. Huang, and K. Griebenow, J. Am. Chem. Soc. 123 (2001).

[28] Y. Levy and O. Becker, J. Chem. Phys. 114, 993 (2001).

[29] Z. Shi, C. Olson, G. Rose, R. Baldwin, and N. Kallenbach, PNAS 99, 9190 (2002). 
[30] A. Garcia, Polymer 45, 669 (2004).

[31] A. Yakubovitch, I. Solov'yov, A. Solov'yov, and W. Greiner, arXiv: physics/0406093

[32] A. Yakubovitch, I. Solov'yov, A. Solov'yov, and W. Greiner, arXiv: physics/0406094

[33] C. Guet, P. Hobza, F. Spiegelman, and F. David, eds., Atomic Clusters and Nanoparticles, NATO Advanced Study Institute, les Houches Session LXXIII, les Houches, 2000 (EDP Sciences and Springer Verlag, Berlin, 2001).

[34] A. Solov'yov and J.-P. Connerade, eds., Latest Advances in Atomic Cluster Collisions Fission, Fusion, Electron, Ion and Photon Impact (World Scientific Press, 2004).

[35] L. Lindgren and J. Morrison, Atomic Many-Body Theory (Springer-Verlag, New York, Heidelberg, Berlin, 1986).

[36] P. Hohenberg and W. Kohn, Phys. Rev. 136, B864 (1964).

[37] A. Becke, Phys.Rev. A 38, 3098 (1988).

[38] C. Lee, W. Yang, and R. Parr, Phys. Rev. B 37, 785 (1988).

[39] R. Parr and W. Yang, Density-Functional Theory of Atoms and Molecules (Oxford University Press, Oxford, New York, 1989).

[40] A. Bax, Prot. Sci. 12, 1 (2003).

[41] S. Sheik, P. Sundararajan, A. Hussain, and K. Sekar, Bioinformatics 18, 1548 (2002).

[42] D. Voet and J. Voet, Biochemistry (John Willey and Sons, Inc., USA, 2004). 